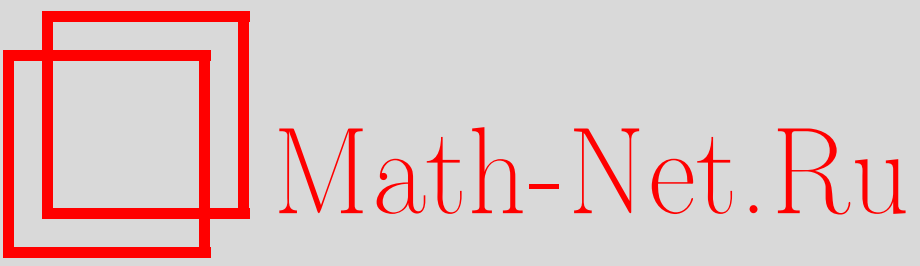

Р. С. Мельник, О. В. Пацаган, Свойства критической точки газ-жидкость симметрической бинарной флюидной смеси, ТМФ, 2000, том 124, номер 2, 339-352

DOI: https://doi.org/10.4213/tmf643

Использование Общероссийского математического портала Math-Net.Ru подразумевает, что вы прочитали и согласны с пользовательским соглашением

http://www.mathnet.ru/rus/agreement

Параметры загрузки:

IP: 52.87 .193 .239

26 апреля 2023 г., 06:17:52 
ТЕОРЕТИЧЕСКАЯ

И МАТЕМАТИЧЕСКАЯ

ФИЗИКА

Том 124, № 2

август, 2000

(C) 2000 г.

Р. С. Мельник*, О.В. Пацаган*

\title{
СВОЙСТВА КРИТИЧЕСКОЙ ТОЧКИ ГАЗ-ЖИДКОСТЬ СИММЕТРИЧЕСКОЙ БИНАРНОЙ ФЛЮИДНОЙ СМЕСИ
}

\begin{abstract}
Предложен микроскопический подход к исследованию поведения симметрической бинарной фоюидной смеси в окрестности критической точки газ-жидкость. Показано, что проблема может быть сведена к расчету статистической суммы трехмерной модели Изинга во внешнем поле. Для симметрической бинарной смеси, частицы которой взаимодействуют с потенциалом прямоугольной ямы, рассчитаны параметры критической точки как функции микроскопического параметра $r$, являющегося мерой относительной силы взаимодействия между частицами одинаковых и различных сортов. Расчеты выполнены для двух областей действия потенциала притяжения: промежуточной $(\lambda=1.5)$ и умеренно дальнодействуюшей $(\lambda=2)$. Результаты расчетов хорошо согласуются с результатами компьютерного моделирования.
\end{abstract}

\section{1. ВВЕДЕНИЕ}

В отличие от чистых компонент бинарные смеси проявляют три различных типа двухфазного равновесия: газ-жидкость, жидкость-жидкость и газ-газ $[1,2]$. Возможность реализации этих явлений и их очередность зависят как от внешних условий, так и от микроскопических параметров смеси. Изучение влияния межчастичных взаимодействий на критические свойства бинарной смеси является интересной и актуальной задачей. На протяжении последнего десятилетия эта задача интенсивно изучалась с помошью методов интегральных уравнений [3-9]. Однако, хотя этот подход и объясняет различные типы фазовых диаграмм в зависимости от микроскопических параметров, он дает только качественную картину рассматриваемого явления. Критические свойства простых флюидов и бинарных смесей изучались с помощью компютерного моделирования методом Монте-Карло [10-14]. В частности, в работе [14] была рассчитана критическая температура газ-жидкость симметрической смеси твердых шаров, взаимодействующих посредством потенциала "прямоугольной ямы”. Поэтому интересно было бы построить теорию с помощью такой простой модели бинарного флюида.

${ }^{*}$ Институт физики конденсированных систем НАН Украины, Львов, Украина. E-mail: romanr @icmp.lviv.ua; oksana@icmp.lviv.ua 
В представленной работе мы предлагаем микроскопический подход к изучению критической точки газ-жидкость бинарной симметрической смеси. Подход основывается на методе коллективных переменных (КП). Этот метод был развит в работе [15] и оказался успешным при изучении фазовых переходов второго рода в трехмерной модели Изинга [16] и критической точки газ-жидкость однокомпонентного флюида [17]. С помощью данного подхода были рассчитаны универсальные и неуниверсальные критические характеристики.

В работе [18] метод КП с выделенной системой отчета был обобщен на случай многокомпонентных непрерьвных систем в большом каноническом ансамбле. С помощью этого подхода в рамках гауссова приближения была изучена фазовая диаграмма симметрической смеси [19-21].

В данной работе мы определяем явную форму эффективного гамильтониана Гинзбурга-Ландау-Вильсона для модели симметрической бинарной смеси в окрестности критической точки газ-жидкость. Далее мы интегрируем выражение для большой статистической суммы, используя метод интегрирования по слоям, предложенный в [16] для трехмерной модели Изинга. В результате этого интегрирования возникают рекуррентные соотношения для коэффициентов гамильтониана Гинзбурга-Ландау-Вильсона, из которых следует уравнение для критической температуры $T_{\mathrm{c}}$. Здесь мы избегаем детального рассмотрения результатов, относяшихся к модели Изинга, отсылая читателя к книге [16], где детально изучена эта проблема. Предлагаемый нами метод дает те же критические индексы, что и в статье [22] (см. таблицу, где приведены показатели критических экспонент и соотношения критических амплитуд трехмерной модели Изинга, полученные в рамках метода КП).

\begin{tabular}{|c|c|c|c|c|c|}
\hline$\nu$ & $\alpha$ & $\beta$ & $\gamma$ & $A^{+} / A^{-}$ & $\Gamma^{+} / \Gamma^{-}$ \\
\hline 0.637 & 0.088 & 0.319 & 1.275 & 0.435 & 6.967 \\
\hline
\end{tabular}

В разделе 2 и дополнении А дается функциональное представление большой статистической суммы. В разделе 3 построена базисная плотность меры (гамильтониан Гинзбурга-Ландау-Вильсона) относительно КП, содержащих параметр порядка. В этом разделе также представлены основные идеи метода интегрирования функционала статистической суммы в окрестности критической точки. В разделе 4 рассчитаны критические характеристики (температура и плотность) бинарной симметрической смеси частиц, взаимодействующих посредством потенциала "прямоугольной ямы”. Проведен анализ полученных результатов, а также их сравнение с результатами компютерного моделирования методом Монте-Карло, полученными в работах $[13,14]$.

\section{2. ФУНКЦИОНАЛЬНОЕ ПРЕДСТАВЛЕНИЕ БОЛЬШОЙ СТАТИСТИЧЕСКОЙ СУММЫ БИНАРНОЙ СМЕСИ}

Рассматривается бинарная флюидная смесь, состоящая из $N_{a}$ частиц сорта "a" и $N_{b}$ частиц сорта " $b$ ". Система находится в объеме $V$ при температуре $T$. Считается, 
что взаимодействие в системе имеет попарно аддитивный характер. Потенциал взаимодействия между частицей сорта $\gamma$, находящейся в точке $\mathbf{r}_{i}$, и частицей сорта $\delta$, находяшейся в точке $\mathbf{r}_{j}$, представляется в виде суммы двух слагаемых

$$
U_{\gamma \delta}\left(\left|\mathbf{r}_{i}-\mathbf{r}_{j}\right|\right)=\Psi_{\gamma \delta}\left(\left|\mathbf{r}_{i}-\mathbf{r}_{j}\right|\right)+\Phi_{\gamma \delta}\left(\left|\mathbf{r}_{i}-\mathbf{r}_{j}\right|\right),
$$

где $\Psi_{\gamma \delta}(r)$ - потенциал короткодействуюшего отталкивания, $\Phi_{\gamma \delta}(r)$ - потенциал притяжения, доминирующий на больших расстояниях.

Функционал большой статистической суммы (БСС) бинарной однородной системы в методе КП с выделенной системой отсчета можно представить в виде произведения двух множителей (см. дополнение А)

$$
\Xi=\Xi_{0} \Xi_{1}
$$

где $\Xi_{0}$ - БСС системы отсчета, которую мы считаем известной. Часть БСС, представленная в фазовом пространстве КП, есть

$$
\begin{aligned}
\Xi_{1}= & \int(d \rho)(d c) \exp \left\{\beta \mu_{1}^{+} \rho_{0}+\beta \mu_{1}^{-} c_{0}-\frac{\beta}{2} \sum_{\mathbf{k}}\left[\widetilde{V}(k) \rho_{\mathbf{k}} \rho_{-\mathbf{k}}+\right.\right. \\
& \left.\left.+2 \widetilde{U}(k) \rho_{\mathbf{k}} c_{\mathbf{k}}+\widetilde{W}(k) c_{\mathbf{k}} c_{-\mathbf{k}}\right]\right\} J(\rho, c) .
\end{aligned}
$$

Химические потенциалы

$$
\mu_{1}^{+}=\frac{1}{\sqrt{2}}\left(\mu_{1}^{a}+\mu_{1}^{b}\right) \quad \text { и } \mu_{1}^{-}=\frac{1}{\sqrt{2}}\left(\mu_{1}^{a}-\mu_{1}^{b}\right)
$$

определяются из условий

$$
\begin{aligned}
& \frac{\partial \ln \Xi_{1}}{\partial\left(\beta \mu_{1}^{+}\right)}=\left\langle N_{a}\right\rangle+\left\langle N_{b}\right\rangle=\langle N\rangle, \\
& \frac{\partial \ln \Xi_{1}}{\partial\left(\beta \mu_{1}^{-}\right)}=\left\langle N_{a}\right\rangle-\left\langle N_{b}\right\rangle .
\end{aligned}
$$

Функции $\widetilde{V}(k), \widetilde{W}(k)$ и $\widetilde{U}(k)$ - это комбинации преобразований Фурье исходных потенциалов взаимодействия $\widetilde{\Phi}_{\gamma \delta}(k)$ :

$$
\begin{aligned}
\widetilde{V}(k) & =\frac{\beta^{-1}}{2}\left[\alpha_{a a}(k)+\alpha_{b b}(k)+2 \alpha_{a b}(k)\right], \\
\widetilde{U}(k) & =\frac{\beta^{-1}}{2}\left[\alpha_{a a}(k)-\alpha_{b b}(k)\right], \\
\widetilde{W}(k) & =\frac{\beta^{-1}}{2}\left[\alpha_{a a}(k)+\alpha_{b b}(k)-2 \alpha_{a b}(k)\right] .
\end{aligned}
$$

Выражение

$$
J(\rho, c)=\int(d \nu)(d \omega) \exp \left\{i 2 \pi \sum_{\mathbf{k}}\left(\omega_{\mathbf{k}} \rho_{\mathbf{k}}+\nu_{\mathbf{k}} c_{\mathbf{k}}\right)+\sum_{n \geqslant 1} \sum_{i_{n} \geqslant 0} D_{n}^{\left(i_{n}\right)}(\omega, \nu)\right\}
$$


- якобиан перехода к КП $\rho_{\mathbf{k}}, c_{\mathbf{k}}$, усредненный по системе отсчета, $\omega_{\mathbf{k}}, \nu_{\mathbf{k}}$ - переменные, сопряженные соответственно к переменным $\rho_{\mathbf{k}}, c_{\mathbf{k}}$. Кумулянтное разложение $D_{n}^{\left(i_{n}\right)}(\omega, \nu)$ имеет вид

$$
\begin{aligned}
D_{n}^{\left(i_{n}\right)}(\omega, \nu)= & {\left[\frac{(-i 2 \pi)^{n}}{n !}\right]\left(\frac{1}{2}\right)^{\frac{n}{2}} \sum_{k_{1} \ldots k_{n}} \mathcal{M}_{n}^{\left(i_{n}\right)}(0, \ldots, 0) \times } \\
& \times \nu_{\mathbf{k}_{1}} \ldots \nu_{\mathbf{k}_{i_{n}}} \omega_{\mathbf{k}_{i_{n}+1}} \ldots \omega_{\mathbf{k}_{n}} \delta_{\mathbf{k}_{1}+\cdots+\mathbf{k}_{n}}
\end{aligned}
$$

Индекс $i_{n}$ обозначает количество переменных $\nu_{\mathbf{k}}$ в кумулянтном разложении (2.7). Кумулянты $\mathcal{M}_{n}^{\left(i_{n}\right)}(0, \ldots, 0)$ являются линейными комбинациями исходных кумулянтов $\mathcal{M}_{\gamma_{1} \ldots \gamma_{n}}(0, \ldots, 0)\left(\gamma_{i}=a, b\right)($ см. дополнение Б).

Рассматривается симметрическая бинарная флюидная смесь (СБФС), т.е. такая смесь, в которой чистые компоненты " $a$ " и " $b$ " идентичны и отличается только взаимодействие меж ду частицами различных сортов. Несмотря на свою простоту СБФС проявляет все три типа фазового равновесия, которые наблюдаются в реальных бинарных флюидах: газ-жидкость, жидкость-жидкость и газ-газ. В случае СБФС $\widetilde{U}(k)=0$ в $(2.3)$ и в кумулянтном разложении (2.7) остаются кумулянты только с парными индексами $i_{n}[20]$.

\section{3. МЕТОД}

Как было показано в работе [20], фазовая диаграмма СБФС состоит из трех областей (рис. 1, где 1 - фазовые переходы газ-газ и газ-жидкость $\left(T_{\mathrm{c}}^{\Gamma-\Gamma}>T_{\mathrm{c}}^{\Gamma-ж}\right) ; 2$ - фазовые переходы газ-жидкость и жидкость-жидкость $\left(T_{\mathrm{c}}^{\Gamma-ж}>T_{\mathrm{c}}^{ж-ж}\right) ; 3$ - только фазовый переход газ-жидкость; $S_{+}$- структурный фактор системы отсчета).

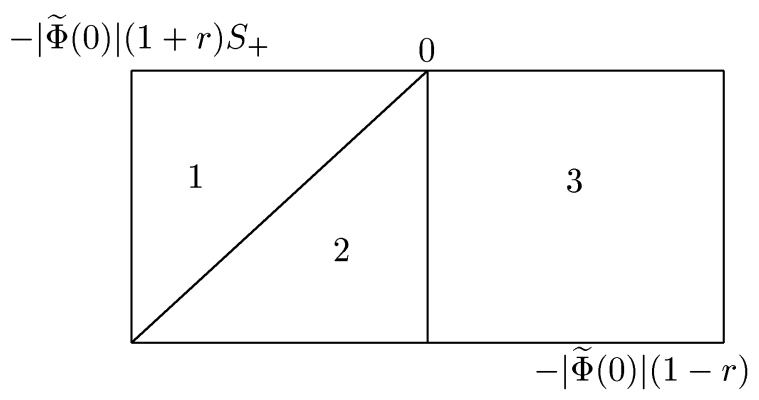

Рис. 1

Очередность фазовых переходов газ-жидкость и смешивание-несмешивание зависит как от внешних условий, так и от микроскопических свойств системы. В такой системе сушествуют две ветви критических температур: ветвь $\left(T_{\mathrm{c}}^{\Gamma-ж}\right)$, связанная с переменной

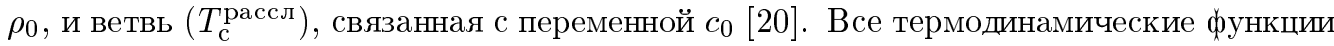
СБФС симметричны относительно концентрации $x=0.5$ и имеют максимум в этой точке $[1,19]$. Концентрация $x=0.5$ является критической для данной модельной смеси. 
В этой работе мы рассматриваем симметрическую флюидную смесь в области 2 или 3 ее фазовой диаграммы.

В случае СБФС переменные $\rho_{0}$ и $c_{0}$ связаны соответственно с параметрами порядка для фазовых переходов газ-жидкость и смешивание-несмешивание [20]. Этот факт разрешает нам разделить КП $\rho_{\mathbf{k}}$ и $c_{\mathbf{k}}$ на основные и вспомогательные в зависимости от того, какой из фазовых переходов рассматривается. Поскольку мы интересуемся критической точкой газ-жидкость, то КП $c_{\mathbf{k}}$ и $\nu_{\mathbf{k}}$ считаем вспомогательными (КП $c_{\mathbf{k}}$ не содержит переменной, связанной с параметром порядка, коэффициенты при второй степени $c_{\mathbf{k}}$ и $\nu_{\mathbf{k}}$ будут отрицательными). Поэтому по $c_{\mathbf{k}}$ и $\nu_{\mathbf{k}}$ можно выполнить интегрирование с гауссовой плотностью меры. Относительно $\rho_{\mathbf{k}}$ как основных КП нужно построить базисное распределение, учитывая высшие степени $\rho_{\mathbf{k}}$ (мы будем рассматривать приближение модели $\left.\rho^{4}\right)$.

Интегрируя в $(2.3)-(2.7)$ по переменным $c_{\mathbf{k}}$ и $\nu_{\mathbf{k}}$, приходим к следуюшему выражению для БСС:

$$
\Xi=\Xi_{0} \Xi_{G}^{c} \int(d \rho) \exp \left\{\beta \mu_{1}^{+} \rho_{0}-\frac{\beta}{2} \sum_{\mathbf{k}} \widetilde{V}(k) \rho_{\mathbf{k}} \rho_{-\mathbf{k}}\right\} J(\rho),
$$

где

$$
\begin{aligned}
\Xi_{G}^{c}= & \prod_{\mathbf{k}} \frac{1}{\sqrt{1+\frac{1}{2} \beta \widetilde{W}(k) \mathcal{M}_{2}^{(2)}(0)}}, \\
J(\rho)= & \int(d \omega) \exp \left\{i 2 \pi \sum_{\mathbf{k}} \omega_{\mathbf{k}} \rho_{\mathbf{k}}+\sum_{n \geqslant 1}^{4} \frac{(-i 2 \pi)^{n}}{n !}\left(\frac{1}{2}\right)^{\frac{n}{2}} \times\right. \\
& \left.\times \sum_{\mathbf{k}_{1} \ldots \mathbf{k}_{n}} \mathcal{M}_{n}(0) \omega_{\mathbf{k}_{1}} \ldots \omega_{\mathbf{k}_{n}} \delta_{\mathbf{k}_{1}+\cdots+\mathbf{k}_{n}}\right\}, \\
& \mathcal{M}_{n}(0)=\mathcal{M}_{n}^{(0)}(0)+\Delta \mathcal{M}_{n},
\end{aligned}
$$

здесь $\Delta \mathcal{M}_{n}$ являются поправками, полученными в результате интегрирования по переменным $c_{\mathbf{k}}$,

$$
\begin{aligned}
\Delta \mathcal{M}_{1}= & \frac{\mathcal{M}_{3}^{(2)}(0)}{12} \frac{1}{\langle N\rangle} \sum_{\mathbf{k}} \tilde{g}(|\mathbf{k}|) \\
\Delta \mathcal{M}_{2}= & \frac{\mathcal{M}_{4}^{(2)}(0)}{12} \frac{1}{\langle N\rangle} \sum_{\mathbf{k}} \tilde{g}(|\mathbf{k}|)+ \\
& +\frac{\left(\mathcal{M}_{3}^{(2)}(0)\right)^{2}}{72} \frac{1}{\langle N\rangle^{2}} \sum_{\mathbf{k}} \tilde{g}(|\mathbf{k}|) \tilde{g}\left(\left|\mathbf{k}_{1}-\mathbf{k}\right|\right) \\
\Delta \mathcal{M}_{3}= & \frac{\mathcal{M}_{3}^{(2)}(0) \mathcal{M}_{4}^{(2)}(0)}{48} \frac{1}{\langle N\rangle^{2}} \sum_{\mathbf{k}} \tilde{g}(|\mathbf{k}|) \tilde{g}\left(\left|\mathbf{k}_{1}-\mathbf{k}\right|\right)+ \\
& +\frac{\left(\mathcal{M}_{3}^{(2)}(0)\right)^{3}}{6} \frac{1}{\langle N\rangle^{3}} \sum_{\mathbf{k}} \tilde{g}(|\mathbf{k}|) \tilde{g}\left(\left|\mathbf{k}_{1}+\mathbf{k}\right|\right) \tilde{g}\left(\left|\mathbf{k}_{2}-\mathbf{k}\right|\right)
\end{aligned}
$$




$$
\begin{aligned}
\Delta \mathcal{M}_{4}= & \frac{\left(\mathcal{M}_{4}^{(2)}(0)\right)^{2}}{96} \frac{1}{\langle N\rangle^{2}} \sum_{\mathbf{k}} \tilde{g}(|\mathbf{k}|) \tilde{g}\left(\left|\mathbf{k}_{1}-\mathbf{k}\right|\right)+ \\
& +\left(\frac{\mathcal{M}_{3}^{(2)}(0)}{3 !}\right)^{4} \frac{1}{\langle N\rangle^{4}} \sum_{\mathbf{k}} \tilde{g}(|\mathbf{k}|) \tilde{g}\left(\left|\mathbf{k}_{1}+\mathbf{k}\right|\right) \times \\
& \times \tilde{g}\left(\left|\mathbf{k}_{2}-\mathbf{k}\right|\right) \tilde{g}\left(\left|\mathbf{k}_{3}+\mathbf{k}_{1}+\mathbf{k}\right|\right),
\end{aligned}
$$

где

$$
\tilde{g}(k)=-\frac{\beta\langle N\rangle \widetilde{W}(k)}{\frac{1}{2} \beta \widetilde{W}(k) \mathcal{M}_{2}^{(2)}(0)+1} .
$$

На рис. 2 изображено типичное поведение потенциала фурье-образа $\widetilde{V}(k) /|\widetilde{V}(0)|$ потенциала $V(r)$. Далее в своих расчетах мы полагаем $\widetilde{V}(k)=0$ для $|\mathbf{k}|>B$. Тогда интегрирование в $(3.1)$ по $\rho_{\mathbf{k}} \mathrm{c}|\mathbf{k}|>B$ приводит к $\delta$-функциям и выражение для $\Xi$ содержит только суммы по $\mathbf{k} \mathrm{c}|\mathbf{k}| \leqslant B$.

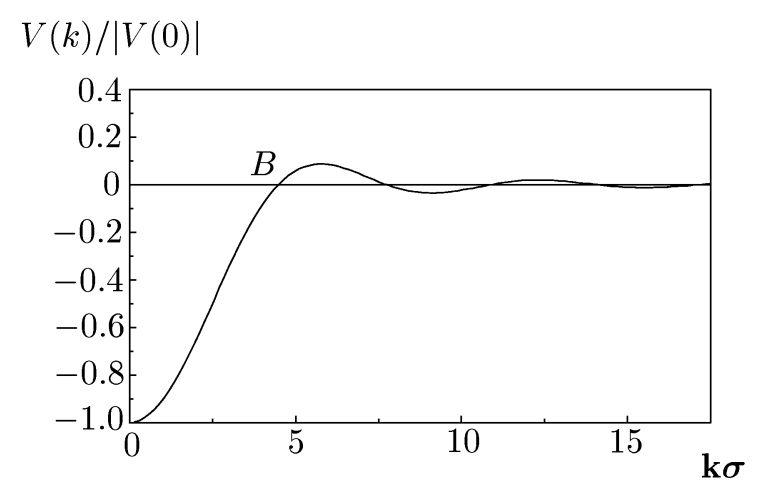

Рис. 2

Мы рассматриваем такой набор векторов $\mathbf{k}(|\mathbf{k}| \leqslant B)$, который отвечает узлам обратной решетки, сопряженной к некоторой прямой блочной решетке $\left\{r_{l}\right\} \mathrm{c}\left\langle N_{B}\right\rangle$ блочными узлами в объеме периодичности $V$ :

$$
\left\langle N_{B}\right\rangle=\frac{V}{C^{3}}=\frac{V}{\left(\frac{\pi}{B}\right)^{3}}=\frac{(B \sigma)^{3}\langle N\rangle}{6 \pi^{2} \eta}
$$

$\eta=\pi \rho \sigma^{3} / 6$ - приведенная плотность. Следовательно, $B$ может считаться величиной первой полузоны Бриллюэна такой решетки.

В результате смешения

$$
\omega_{\mathbf{k}}=\omega_{\mathbf{k}}^{\prime}+\Delta \delta_{\mathbf{k}}, \quad \rho_{\mathbf{k}}=\rho_{\mathbf{k}}^{\prime}+\widetilde{\mathcal{M}}_{1} \delta_{\mathbf{k}}
$$


где

$$
\begin{gathered}
\Delta=-\frac{i}{2 \pi} \frac{\overline{\mathcal{M}}_{3}(0)}{\overline{\mathcal{M}}_{4}(0)} \delta_{\mathbf{k}}, \\
\widetilde{\mathcal{M}}_{1}=\overline{\mathcal{M}}_{1}(0)-\frac{\overline{\mathcal{M}}_{2}(0) \overline{\mathcal{M}}_{3}(0)}{\overline{\mathcal{M}}_{4}(0)}+\frac{\overline{\mathcal{M}}_{3}^{3}(0)}{3 \overline{\mathcal{M}}_{4}^{2}(0)}
\end{gathered}
$$

$\left(\overline{\mathcal{M}}_{n}(0)=\mathcal{M}_{n}(0) /(\sqrt{2})^{n}, n=1, \ldots, 4\right)$, величина $\Xi$ преобразуется к виду, содержашему только слагаемые с $\widetilde{\mathcal{M}}_{1}(0), \widetilde{\mathcal{M}}_{2}(0)$ и $\widetilde{\mathcal{M}}_{4}(0)$ (штрихи у $\rho_{\mathbf{k}}$ и $\omega_{\mathbf{k}}$ опушены):

$$
\begin{aligned}
\Xi= & \Xi_{0} \Xi_{G}^{(1)} \int \exp \left\{\mu^{*} \rho_{0}-\frac{\beta}{2} \sum_{\mathbf{k}<B} \tilde{V}(k) \rho_{\mathbf{k}} \rho_{-\mathbf{k}}+\right. \\
& +i 2 \pi \sum_{\mathbf{k}<B} \omega_{\mathbf{k}} \rho_{\mathbf{k}}-\frac{(2 \pi)^{2}}{2} \widetilde{\mathcal{M}}_{2}(0) \sum_{\mathbf{k}<B} \omega_{\mathbf{k}} \omega_{-\mathbf{k}}-\frac{(2 \pi)^{4}}{4 !\left\langle N_{B}\right\rangle}\left|\widetilde{\mathcal{M}}_{4}(0)\right| \times \\
& \left.\times \sum_{\mathbf{k}_{1} \ldots \mathbf{k}_{4}<B} \omega_{\mathbf{k}_{1}} \omega_{\mathbf{k}_{2}} \omega_{\mathbf{k}_{3}} \omega_{\mathbf{k}_{4}} \delta_{\mathbf{k}_{1}+\cdots+\mathbf{k}_{4}}\right\}(d \omega)^{N_{B}}(d \rho)^{N_{B}},
\end{aligned}
$$

где

$$
\begin{aligned}
\Xi_{G}^{(1)}= & \Xi_{G}^{c} \exp \left\{\mu^{*} \widetilde{\mathcal{M}}_{1}+\frac{\beta \widetilde{V}^{*}(0)}{2} \widetilde{\mathcal{M}}_{1}^{2}-\right. \\
& \left.-\frac{\overline{\mathcal{M}}_{1}(0) \overline{\mathcal{M}}_{3}(0)}{\overline{\mathcal{M}}_{4}(0)}-\frac{\overline{\mathcal{M}}_{2}(0) \overline{\mathcal{M}}_{3}^{2}(0)}{2 \overline{\mathcal{M}}_{4}^{2}(0)}-\frac{\overline{\mathcal{M}}_{3}^{4}(0)}{8 \overline{\mathcal{M}}_{4}^{3}(0)}\right\}, \\
\mu^{*}= & h-a_{1}, \quad a_{1}=\frac{\overline{\mathcal{M}}_{3}(0)}{\left|\overline{\mathcal{M}}_{4}(0)\right|}+\beta \widetilde{V}^{*}(0) \widetilde{\mathcal{M}}_{1}, \quad h=\beta \mu_{1}^{+}, \\
\widetilde{\mathcal{M}}_{2}(0)= & \overline{\mathcal{M}}_{2}(0)-\frac{\overline{\mathcal{M}}_{3}^{2}(0)}{2 \overline{\mathcal{M}}_{4}(0)}, \\
\widetilde{\mathcal{M}}_{4}(0)= & \left\langle N_{B}\right\rangle \overline{\mathcal{M}}_{4}(0) .
\end{aligned}
$$

Выражение (3.8) для $\Xi$ имеет такую же форму, как и выражение для статистической суммы трехмерной модели Изинга в постоянном внешнем поле $\left(a_{1}-\beta \mu_{1}^{+}\right)$с той лиш разницей, что кумулянты $\widetilde{\mathcal{M}}_{2}(0), \widetilde{\mathcal{M}}_{4}(0)$ в $(3.8)$ являются функциями приведенной плотности $\eta$, температуры $T$ и параметров потенциала притяжения $\widetilde{\Phi}_{\gamma \delta}(k)$.

После интегрирования по $\omega_{\mathbf{k}}$ получаем следующую форму для БСС:

$$
\Xi=\Xi_{0} \Xi_{G}^{(1)}\left[Z\left(\widetilde{\mathcal{M}}_{2}, \widetilde{\mathcal{M}}_{4}\right)\right]^{\left\langle N_{B}\right\rangle}(\sqrt{2})^{\left\langle N_{B}\right\rangle-1} \int \exp \left[E_{4}(\rho)\right](d \rho)^{N_{B}}
$$

здесь

$$
\begin{aligned}
E_{4}(\rho)= & \mu^{*} \rho_{0}-\frac{1}{2} \sum_{\mathbf{k}<B} d_{2}(k) \rho_{\mathbf{k}} \rho_{-\mathbf{k}}-\frac{a_{4}}{4 !\left\langle N_{B}\right\rangle} \times \\
& \times \sum_{\mathbf{k}_{1} \ldots \mathbf{k}_{4}<B} \rho_{\mathbf{k}_{1}} \ldots \rho_{\mathbf{k}_{4}} \delta_{\mathbf{k}_{1}+\cdots+\mathbf{k}_{4}}+\cdots
\end{aligned}
$$


И

$$
\begin{aligned}
& Z\left(\widetilde{\mathcal{M}}_{2}, \widetilde{\mathcal{M}}_{4}\right)=\left(\frac{1}{2 \pi}\right)^{\frac{1}{2}}\left(\frac{3}{\left|\widetilde{\mathcal{M}}_{4}(0)\right|}\right)^{\frac{1}{4}} e^{\frac{x^{2}}{4}} U(0, x), \\
& d_{2}(k)=a_{2}+\beta \widetilde{V}(k), \quad a_{2}=\sqrt{\frac{3}{\left|\widetilde{\mathcal{M}}_{4}(0)\right|}} K(x), \quad a_{4}=\frac{3}{\left|\widetilde{\mathcal{M}}_{4}(0)\right|} L(x),
\end{aligned}
$$

где

$$
\begin{gathered}
K(x)=\frac{U(1, x)}{U(0, x)}, \\
L(x)=3 K^{2}(x)+2 x K(x)-2, \\
x=\sqrt{\frac{3}{\left|\widetilde{\mathcal{M}}_{4}(0)\right|} \widetilde{\mathcal{M}}_{2}(0) .}
\end{gathered}
$$

$U(a, x)$ - функция параболического цилиндра Вебера [23]. Выражения (3.10)-(3.13) аналогичны по форме выражениям, полученным в работе [17] для однокомпонентной системы. Такое совпадение - результат симметричности модели. $E_{4}(\rho)$ - гамильтониан Гинзбурга-Ландау-Вильсона для СБФС в окрестности критической точки газ-жидкость.

Для того чтобы проинтегрировать БСС (3.10)-(3.13) по $\rho_{\mathbf{k}}$ и определить уравнение для критической температуры, используем метод, предложенный в работах $[16,24]$ для модели Изинга. Суть этого метода заключается в поэтапном интегрировании по слоям фазового пространства КП начиная с переменных $\rho_{\mathbf{k}}$, которые отвечают коротковолновым флуктуациям. Изменение коэффициентов в $E_{4}(\rho)$, которое происходит в результате интегрирования по $\rho_{\mathbf{k}}$ в $n$ последовательных слоях фазового пространства КП, описывается рекуррентной формулой, полученной в работе [16]. В случае $T>T_{\mathrm{c}}$ в интервале $[0, B]$ существуют три характерные области [16]. Первая область $B_{m_{\tau}}<k \leqslant B$ отвечает сильно коррелируюшим флуктуациям $\rho_{\mathbf{k}}$. Соответствуюшая им плотность меры негауссова. Здесь применяется процедура, основанная на симметрии ренормализационной группы. Это - область критического режима. Вторая область $0<k \leqslant B_{m_{\tau}}$ отвечает флуктуациям, учитываюшимся с гауссовой плотностью меры. Это - граничный гауссов режим. Третья область содержит лишь одну точку $k=0$. Переменная $\rho_{0}-$ макроскопическая величина, связанная с флуктуациями плотности числа частиц во "внешнем поле" $\mu^{*}$.

Интегрирование в (3.10) проводится по следующей схеме [16]. Область $(0, B)$ разбивается на интервалы $\left(B_{1}, B\right), \ldots,\left(B_{i+1}, B_{i}\right), \ldots$, где $B_{n}=B / S^{n}(S$ - параметр деления). Каждому интервалу соответствует слой индексов $\mathbf{k}$ в зоне Бриллюэна. Каждому слою индексов $\mathbf{k}$ соответствует слой в фазовом пространстве $\rho_{\mathbf{k}}$. Поэтапно интегрируя по слоям, мы получаем набор блочных решеток с соответственно возрастаюшими периодами блоков и с соответствующими каждой блочной решетке гамильтонианами. Каждый гамильтониан характеризуется коэффициентами $d_{2}, a_{4} ; d_{2}^{(1)}, a_{4}^{(1)} ; d_{2}^{(2)}, a_{4}^{(2)}$ и т.д. Для последовательности блочных гамильтонианов $\left\{d_{2}^{(n)}, a_{4}^{(n)}\right\}$ имеют место симметрия ренормализационной группы и неподвижная точка седлового типа. Поскольку 
выражения для исходных коэффициентов $d(k)$ и $a_{4}$ известны (см. (3.11)-(3.13)), то ренормгрупповые решения будут также функциями микроскопических параметров, плотности и температуры.

Критический режим имеет место для всех переменных $\rho_{\mathbf{k}}$ в критической точке. Поэтому критическую температуру можно определить из решения рекуррентных соотношений. Используя результаты, полученные в [24], приходим к выражению

$$
A\left(\beta_{\mathrm{c}} \tilde{V}(0)\right)^{2}+B \beta_{\mathrm{c}} \tilde{V}(0)+D=0
$$

где

$$
\begin{gathered}
A=1-f_{0}-R^{(0)} \sqrt{\varphi_{0}}, \\
B=-a_{2}, \\
D=\frac{a_{4} R^{(0)}}{\sqrt{\varphi_{0}}},
\end{gathered}
$$

$f_{0}, \varphi_{0}$ - координаты приведенной неподвижной точки, $R^{(0)}$ - универсальная функция параметра $S$. В случае приближения модели $\rho^{4}$ оптимальное значение параметра деления фазового пространства на слои $S^{*}=3.4252$. Это значение определяется из условия обращения в нуль коэффициента $d_{2}^{(n)}(0)$ в неподвижной точке [16]. Значения величин $f_{0}$, $\varphi_{0}, R^{(0)}$, соответствуюшие $S=S^{*}$, взяты из работы [24]. Из условия $\mu^{*}=0$ [17] мы получаем уравнение

$$
\mathcal{M}_{3}(0)=0
$$

которое дает возможность определить критическую плотность системы.

\section{4. РЕЗУЛЬТАТЫ И ОБСУЖДЕНИЯ}

В этом разделе представлены результаты расчетов критической точки газ-жидкость симметрической смеси, полученные с помошью предложенного нами метода. Здесь также проведено сравнение наших результатов с недавними результатами компютерного моделирования методом Монте-Карло [13, 14].

Рассматриваемая система - это симметрическая бинарная смесь твердых шаров, взаимодействующих посредством потенциала

$$
U_{\gamma \delta}(r)= \begin{cases}\infty, & r<\sigma, \\ -\epsilon_{\gamma \delta}, & \sigma \leqslant r<\lambda \sigma \\ 0, & r \geqslant \lambda \sigma\end{cases}
$$

где $\sigma$ - диаметр твердых шаров, $\lambda$ - область действия потенциала, $\epsilon_{\gamma} \delta$ - глубина потенциальной ямы. Для симметрической смеси $\epsilon_{a a}=\epsilon_{b b}=\epsilon \neq \epsilon_{a b}$. В данной работе мы выбираем $\lambda=1.5$ и $\lambda=2.0$, поскольку именно для этих значений $\lambda$ имеются результаты компьютерного моделирования методом Монте-Карло. 
Используя разбиение Викса-Чандлера-Андерсена [25], мы разделяем потенциал $U_{\gamma \delta}(r)$ на коротко- и дальнодействуюшие части:

$$
\begin{aligned}
& \Psi_{\gamma \delta}(r)= \begin{cases}\infty, & r \leqslant \sigma, \\
0, & r>\sigma,\end{cases} \\
& \Phi_{\gamma \delta}(r)= \begin{cases}-\epsilon_{\gamma \delta}, & 0 \leqslant r \leqslant \lambda \sigma, \\
0, & r>\lambda \sigma .\end{cases}
\end{aligned}
$$

В нашем случае системой отсчета является система твердых шаров диаметра $\sigma$ (см. (4.1)). Фурье-преобразование функции (4.2) имеет вид

$$
\widetilde{\Phi}_{\gamma \delta}(k)=\widetilde{\Phi}_{\gamma \delta}(0) \frac{3}{(\lambda x)^{3}}[-\lambda x \cos (\lambda x)+\sin (\lambda x)]
$$

где

$$
x=k \sigma, \quad \widetilde{\Phi}_{\gamma \delta}(0)=-\epsilon_{\gamma \delta} \sigma^{3} \frac{4 \pi}{3} \lambda^{3}
$$

Формулы, по которым рассчитаны кумулянты $\mathcal{M}_{n}^{\left(i_{n}\right)}(0, \ldots, 0)$, приведены в дополнении Б. Для величины $S_{2}(0)$ использовано приближение Перкуса-Йевика

$$
S_{2}(0)=\frac{(1-\eta)^{4}}{(1+2 \eta)^{2}}
$$

Уравнения (3.14), (3.15) решаются численно с помошью самосогласованной процедуры, учитываюшей зависимость коэффициентов $a_{2}$ и $a_{4}\left(\right.$ так же как и кумулянтов $\left.\mathcal{M}_{n}(0)\right)$ от $\beta_{\mathrm{c}}$.

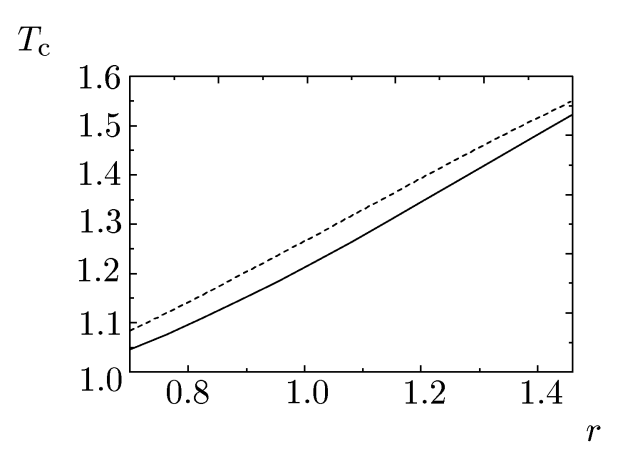

a

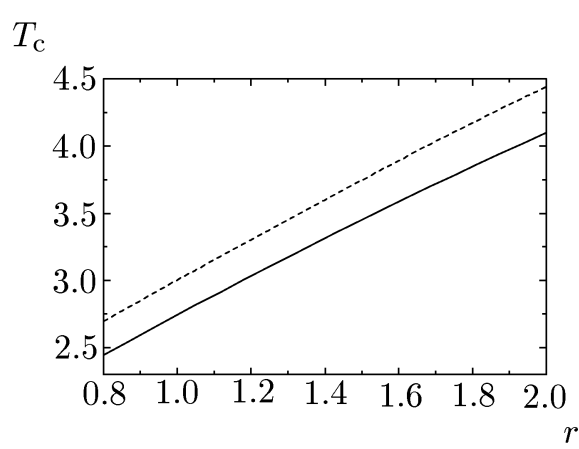

б

Рис. 3

На рис. 3 показана зависимость критической температуры газ-жидкость $T_{\mathrm{c}}\left(T_{\mathrm{c}}=\right.$ $\left.k_{\mathrm{B}} T / \epsilon\right)$ от микроскопического параметра $r=\epsilon_{a b} / \epsilon$, характеризующего относительную силу взаимодействия между частищами одинаковых и различных сортов при $\lambda=1.5$ (рис. 3а) и $\lambda=2.0$ (рис. 3б), сплошная линия - приближение модели $\rho^{4}$, штриховая линия - гауссово приближение. 


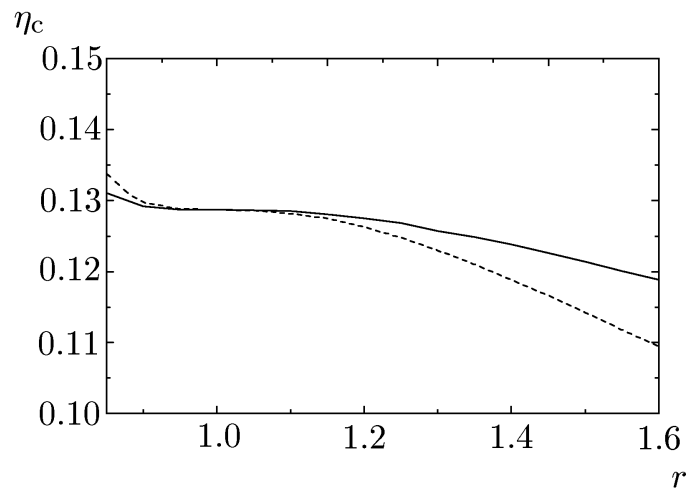

Рис. 4

На рис. 4 изображена зависимость критической плотности $\eta_{\mathrm{c}}$ от $r$ при $\lambda=1.5$ (штриховая линия) и $\lambda=2.0$ (сплошная линия). Сравним наши результаты с результатами компьютерного моделирования методом Монте-Карло. При $\lambda=1.5$ и $r=0.72$ мы получаем $T_{\mathrm{c}}=1.055$, тогда как метод Монте-Карло дает $T_{\mathrm{c}}=1.06(1)$ [14]. При $\lambda=2.0$ и $r=1.0$ (случай однокомпонентной системы) мы получаем $T_{\mathrm{c}}=2.753$ и $\eta_{\mathrm{c}}=0.129$, тогда как компьютерное моделирование дает $T_{\mathrm{c}}=2.684(51)$ и $\eta_{\mathrm{c}}=0.123(43)$ [13].

\section{5. ВЫВОДЫ}

В этой работе предложен метод для изучения зависимости параметров критической точки газ-жидкость симметрической бинарной флюидной смеси от ее микроскопических свойств. Используя этот метод, мы рассчитали зависимость критической температуры и критической плотности бинарной смеси твердых шаров, взаимодействующих через потенциал "прямоугольной ямы", от микроскопическогопараметра $r$, который является мерой относительного взаимодействия между частишами одинаковых и различных сортов. Наши результаты хорошо согласуются с результатами компютерного моделирования методом Монте-Карло. Свои результаты мы можем улучшить, как принимая во внимание область $\mathbf{k} \mathbf{c}|\mathbf{k}|>B$ (см. рис. 2), так и используя приближение более высокого порядка чем $\rho^{4}$.

ДОПОЛНЕНИЕ А

Выражение для БСС двухкомпонентной флюидной смеси в представлении КП с выделенной системой отсчета может быть записано [18] в виде

$$
\Xi=\Xi_{0} \Xi_{1}
$$

где

$$
\Xi_{0}=\sum_{N_{a}=0}^{\infty} \sum_{N_{b}=0}^{\infty} \prod_{\gamma=a}^{b} \frac{\exp \left(\beta \mu_{0}^{\gamma} N_{\gamma}\right)}{N_{\gamma} !} \int(d \Gamma) \exp \left[-\frac{\beta}{2} \sum_{\gamma, \delta=a, b} \sum_{i, j} \psi_{\gamma \delta}\left(r_{i j}\right)\right]
$$


- БСС системы отсчета; $\beta=1 / k_{\mathrm{B}} T, k_{\mathrm{B}}$ - постоянная Больцмана, $T$ - температура; $(d \Gamma)=\prod_{a, b} d \Gamma_{N_{\gamma}}, d \Gamma_{N_{\gamma}}=d \mathbf{r}_{1}^{\gamma} d \mathbf{r}_{2}^{\gamma} \ldots d \mathbf{r}_{N_{\gamma}}^{\gamma}$ - элемент объема фазового пространства частиц сорта $\gamma ; \mu_{0}^{\gamma}$ - химический потенциал частиц сорта $\gamma$ в системе отсчета.

Часть БСС, определенная в фазовом пространстве КП, имеет форму функционального интеграла:

$$
\Xi_{1}=\int(d \rho) \exp \left[\beta \sum_{\gamma} \mu_{1}^{\gamma} \rho_{0, \gamma}-\frac{1}{2 \beta} \sum_{\gamma, \delta=a, b} \sum_{\mathbf{k}} \alpha_{\gamma \delta}(k) \rho_{\mathbf{k}, \gamma} \rho_{-\mathbf{k}, \delta}\right] J\left(\rho_{a}, \rho_{b}\right),
$$

где $\mu_{1}^{\gamma}$ - часть химического потенциала частиц сорта $\gamma$ :

$$
\mu_{1}^{\gamma}=\mu_{\gamma}-\mu_{0}^{\gamma}+\frac{1}{2 \beta} \sum_{\mathbf{k}} \alpha_{\gamma \gamma}(k)
$$

которая определяется из уравнения

$$
\frac{\partial \ln \Xi_{1}}{\partial \beta \mu_{1}^{\gamma}}=\left\langle N_{\gamma}\right\rangle
$$

$\mu_{\gamma}-$ полный химический потенциал частиц сорта $\gamma$,

$$
\alpha_{\gamma \delta}(k)=\frac{\beta}{V} \tilde{\phi}_{\gamma \delta}(k)
$$

$\left\langle N_{\gamma}\right\rangle$ - среднее число частиц сорта $\gamma$. Переменные $\rho_{\mathbf{k}, \gamma}=\rho_{\mathbf{k}, \gamma}^{c}-i \rho_{\mathbf{k}, \gamma}^{s}(\gamma=a, b)$ в формуле (А.1) - коллективные переменные сорта $\gamma$, индексы $c$ и $s$ обозначают действительную и мнимую части $\rho_{\mathbf{k}, \gamma} ; \rho_{\mathbf{k}, \gamma}^{c}$ и $\rho_{\mathbf{k}, \gamma}^{s}$ описывают величину $\mathbf{k}$-й моды фолуктуаций числа частищ сорта $\gamma$. Каждая из величин $\rho_{\mathbf{k}, \gamma}^{c}$ и $\rho_{\mathbf{k}, \gamma}^{s}$ принимает все действительные значения от $-\infty$ до $+\infty,(d \rho)$ - элемент объема фазового пространства КП:

$$
(d \rho)=\prod_{\gamma} d \rho_{0, \gamma} \prod_{\mathbf{k} \neq 0}^{\prime} d \rho_{\mathbf{k}, \gamma}^{c} d \rho_{\mathbf{k}, \gamma}^{s}
$$

Штрих возле знака произведения обозначает, что $\mathbf{k}$ изменяется в верхнем полупространстве. Величина $J\left(\rho_{a}, \rho_{b}\right)$ есть якобиан перехода к КП, усредненньй по системе отсчета,

$$
\begin{aligned}
J\left(\rho_{a}, \rho_{b}\right)= & \int(d \nu) \prod_{\gamma=a}^{b} \exp \left[i 2 \pi \sum_{\vec{k}} \nu_{\mathbf{k}, \gamma} \rho_{\mathbf{k}, \gamma}\right] \times \\
& \times \exp \left[\sum_{n \geqslant 1} \frac{(-i 2 \pi)^{n}}{n !} \sum_{\gamma_{1} \ldots \gamma_{n}} \sum_{\mathbf{k}_{1} \ldots \mathbf{k}_{n}} M_{\gamma_{1} \ldots \gamma_{n}}\left(\mathbf{k}_{1}, \ldots, \mathbf{k}_{n}\right) \nu_{\mathbf{k}_{1}, \gamma_{1}} \ldots \nu_{\mathbf{k}_{n}, \gamma_{n}}\right]
\end{aligned}
$$

где переменные $\nu_{\mathbf{k}, \gamma}$ - сопряженные к $\mathrm{K} \rho_{\mathbf{k}, \gamma} ; M_{\gamma_{1} \ldots \gamma_{n}}\left(\mathbf{k}_{1}, \ldots, \mathbf{k}_{n}\right)-n$-й кумулянт, связанный с $n$-частичным структурным фактором системы отсчета $S_{\gamma_{1} \ldots \gamma_{n}}\left(k_{1}, \ldots, k_{n}\right)$ соотношением

$$
M_{\gamma_{1} \ldots \gamma_{n}}\left(\mathbf{k}_{1}, \ldots, \mathbf{k}_{n}\right)=\sqrt[n]{N_{\gamma_{1}} \ldots N_{\gamma_{n}}} S_{\gamma_{1} \ldots \gamma_{n}}\left(k_{1}, \ldots, k_{n}\right) \delta_{\mathbf{k}_{1}+\cdots+\mathbf{k}_{n}}
$$


где $\delta_{\mathbf{k}_{1}+\cdots+\mathbf{k}_{n}}-$ символ Кронекера.

Кумулянт $M_{\gamma_{1} \ldots \gamma_{n}}\left(\mathbf{k}_{1}, \ldots, \mathbf{k}_{n}\right)$ имеет сложную зависимость от волновых векторов $\mathbf{k}_{1}, \ldots, \mathbf{k}_{n}$. Здесь и далее величину $M_{\gamma_{1} \ldots \gamma_{n}}\left(\mathbf{k}_{1}, \ldots, \mathbf{k}_{n}\right)$ заменяем ее значением в длинноволновом пределе $M_{\gamma_{1} \ldots \gamma_{n}}(0, \ldots, 0)$. Функция $\tilde{\phi}_{\gamma \delta}(k)$ есть преобразование Фурье потенциала притяжения $\phi_{\gamma \delta}(r)$. Она отрицательна при малых $\mathbf{k}$ и $\lim _{\mathbf{k} \rightarrow \infty} \tilde{\phi}_{\gamma \delta}(k)=0$.

В формуле (А.1) мы переходим к КП $\rho_{\mathbf{k}}$ и $c_{\mathbf{k}}$ (соответственно к $\omega_{\mathbf{k}}$ и $\gamma_{\mathbf{k}}$ ) с помошью ортогонального линейного преобразования

$$
\begin{array}{ll}
\rho_{\mathbf{k}}=\frac{\sqrt{2}}{2}\left(\rho_{\mathbf{k}, a}+\rho_{\mathbf{k}, b}\right), & c_{\mathbf{k}}=\frac{\sqrt{2}}{2}\left(\rho_{\mathbf{k}, a}-\rho_{\mathbf{k}, b}\right), \\
\omega_{\mathbf{k}}=\frac{\sqrt{2}}{2}\left(\nu_{\mathbf{k}, a}+\nu_{\mathbf{k}, b}\right), & \nu_{\mathbf{k}}=\frac{\sqrt{2}}{2}\left(\nu_{\mathbf{k}, a}-\nu_{\mathbf{k}, b}\right) .
\end{array}
$$

Теперь $\rho_{\mathbf{k}}$ и $c_{\mathbf{k}}$ связаны соответственно с модами флуктуаций общей плотности частиц и относительной плотности (или концентрации) частиц.

Окончательно для $\Xi_{1}$ получаем представление в виде соотношений $(2.3)-(2.7)$.

ДОПОЛНЕНИЕ Б

Кумулянты $\mathcal{M}_{n}^{\left(i_{n}\right)}(0)$ с $n \leqslant 4$ выражаются через исходные кумулянты $\mathcal{M}_{\gamma_{1} \ldots \gamma_{n}}(0, \ldots$ $\ldots, 0)\left(\gamma_{1}, \ldots, \gamma_{n}=a, b\right)$ следуюшим образом [20]:

$$
\begin{aligned}
\mathcal{M}_{1}^{(0)}(0)= & \mathcal{M}_{a}(0)+\mathcal{M}_{b}(0)=\langle N\rangle \\
\mathcal{M}_{1}^{(1)}(0)= & \mathcal{M}_{a}(0)-\mathcal{M}_{b}(0)=\left\langle N_{a}\right\rangle-\left\langle N_{b}\right\rangle, \\
\mathcal{M}_{2}^{(0)}(0)= & \mathcal{M}_{a a}(0)+\mathcal{M}_{b b}(0)+2 \mathcal{M}_{a b}(0), \\
\mathcal{M}_{2}^{(1)}(0)= & \mathcal{M}_{a a}(0)-\mathcal{M}_{b b}(0) \\
\mathcal{M}_{2}^{(2)}(0)= & \mathcal{M}_{a a}(0)+\mathcal{M}_{b b}(0)-2 \mathcal{M}_{a b}(0), \\
\mathcal{M}_{3}^{(0)}(0)= & \mathcal{M}_{a a a}(0)+\mathcal{M}_{b b b}(0)+3\left[\mathcal{M}_{a a b}(0)+\mathcal{M}_{a b b}(0)\right], \\
\mathcal{M}_{3}^{(1)}(0)= & \mathcal{M}_{a a a}(0)-\mathcal{M}_{b b b}(0)+\mathcal{M}_{a a b}(0)-\mathcal{M}_{a b b}(0), \\
\mathcal{M}_{3}^{(2)}(0)= & \mathcal{M}_{a a a}(0)+\mathcal{M}_{b b b}(0)-\mathcal{M}_{a a b}(0)-\mathcal{M}_{a b b}(0), \\
\mathcal{M}_{3}^{(3)}(0)= & \mathcal{M}_{a a a}(0)-\mathcal{M}_{b b b}(0)-3\left[\mathcal{M}_{a a b}(0)-\mathcal{M}_{a b b}(0)\right], \\
\mathcal{M}_{4}^{(0)}(0)= & \mathcal{M}_{a a a a}(0)+\mathcal{M}_{b b b b}(0)+ \\
& +4\left[\mathcal{M}_{a a a b}(0)+\mathcal{M}_{a b b b}(0)\right]+6 \mathcal{M}_{a a b b}(0), \\
\mathcal{M}_{4}^{(1)}(0)= & \mathcal{M}_{a a a a}(0)-\mathcal{M}_{b b b b}(0)+2\left[\mathcal{M}_{a a a b}(0)-\mathcal{M}_{a b b b}(0)\right], \\
\mathcal{M}_{4}^{(2)}(0)= & \mathcal{M}_{a a a a}(0)+\mathcal{M}_{b b b b}(0)-2 \mathcal{M}_{a a b b}(0), \\
\mathcal{M}_{4}^{(3)}(0)= & \mathcal{M}_{a a a a}(0)-\mathcal{M}_{b b b b}(0)-2\left[\mathcal{M}_{a a a b}(0)-\mathcal{M}_{a b b b}(0)\right], \\
\mathcal{M}_{4}^{(4)}(0)= & \mathcal{M}_{a a a a}(0)+\mathcal{M}_{b b b b}(0)- \\
& -4\left[\mathcal{M}_{a a a b}(0)+\mathcal{M}_{a b b b}(0)\right]+6 \mathcal{M}_{a a b b}(0) .
\end{aligned}
$$

Такие же соотношения справедливы при $\mathbf{k}_{i} \neq 0$. 
$N$-й кумулянт $\mathcal{M}_{n}^{\left(i_{n}\right)}(0)$ с $i_{n}=0$ связан с $n$-м структурным фактором однокомпонентной системы $S_{n}(0)[20]$,

$$
\mathcal{M}_{n}^{(0)}(0)=\langle N\rangle S_{n}(0)
$$

Структурные факторы $S_{n}(0)(n \geqslant 2)$ выражаются через $S_{2}(0)$ с помощью цепочки уравнений для корреляционных функций $[26,27]$. Кумулянты с $i_{n} \neq 0$ выражаются через $\mathcal{M}_{n}^{(0)}(0)$ (см. формулу (4.8) в [20]).

\section{Список литературы}

[1] P. H. Van Konynenburg, R. L. Scott. Philos. T. Roy. Soc. A. 1980. V. 298. P. 1442.

[2] J.S. Rowlinson, F. L. Swinton. Liquids and Liquid Mixtures. London: Butterworth, 1982.

[3] M. C. Abramo, C. Caccamo, G. Giunta. Phys. Rev. A. 1986. V. 34. P. 3279.

[4] D. B. McGuigan, P. A. Monson. Mol. Phys. 1987. V. 62. P. 3.

[5] C. Caccamo, G. Malescio. Phys. Rev. A. 1989. V. 40. P. 6384.

[6] G. Malescio. Phys. Rev. A. 1990. V. 42. P. 6241.

[7] X.S. Chen, F. Forstmann. J. Chem. Phys. 1992. V. 97. P. 3696.

[8] G. Malescio. J. Chem. Phys. 1992. V. 96. P. 648.

[9] C. Caccamo, G. Giunta. Mol. Phys. 1993. V. 78. P. 83.

[10] J. R. Recht, A.Z. Panagiotopoulos. Mol. Phys. 1993. V. 80. P. 843.

[11] D. G. Green, G. Jackson, E. de Miguel, L.R. Rull. J. Chem. Phys. 1994. V. 101. P. 3190.

[12] N. B. Wilding. Phys. Rev. E. 1997. V. 55. P. 6627.

[13] E. de Miguel. Phys. Rev. E. 1997. V. 55. P. 1347.

[14] N. B. Wilding. Phys. Rev. E. 1998. V. 58. P. 2201.

[15] И.Р. Юхновский, М. Ф. Головко. Статистическая теория классических равновесных систем. Киев: Наукова думка, 1980.

[16] И. Р. Юхновский. Фазовые переходы второго рода. Метод коллективных переменных. Киев: Наукова думка, 1985.

[17] I. R. Yukhnovskii, V. O. Kolomiets, I. M. Idzyk. J. Stat. Phys. 1995. V. 80. P. 405.

[18] О. В. Пацаган, И. Р. Юхновский. ТМФ. 1990. Т. 83. № 1. С. 72.

[19] O. V. Patsahan. Cond. Matt. Phys. 1995. № 5. P. 124.

[20] I. R. Yukhnovskii, O. V. Patsahan. J. Stat. Phys. 1995. V. 81. P. 647.

[21] О. В. Пачаган. УФЖ. 1996. Т. 41. С. 877.

[22] M. P. Kozlovskii, I. V. Pylyuk, V. V. Duhovii. Cond. Mat. Phys. 1997. № 11. P. 17.

[23] М. Абрамовии, И. Стиган. Справочник по специальным функциям. М.: Мир, 1979.

[24] М. П. Козловський, І. Р. Юхновсъкий. Метод самоузгодженого опису критичнӧ̈ поведінки тривимірних ізінгоподібних систем на мікроскопічному рівні. Препринт ІФКС-97-25У. Львів: Інститут фізики конденсованих систем, 1997.

[25] J. D. Weeks, D. Chandler, H. C. Andersen. J. Chem. Phys. 1971. V. 54. P. 12.

[26] И. Р. Юхновский, И. М. Идзик. Термодинамический предел вблизи критической точки жидкость-пар. Препринт ИТФ-85-97Р. Киев: Институт теоретической физики, 1985.

[27] G. Stell. Cluster expansions for classical systems in equilibrium. In: The Equilibrium Theory of Classical Fluids. Eds. H.L. Frish, J.L. Lebowitz. New York: Benjamin, 1964. P. 171.

Поступила в редакцию 8.XII.1999 г., после доработки 24.III.2000 г. 